MAURICIO PÉREZ ABRIL*

\title{
LA INVESTIGACIÓN SOBRE LA PROPIA PRÁCTICA COMO ESCENARIO DE CAMBIO ESCOLAR**
}

\begin{abstract}
Resumen
El presente texto de reflexión aborda dos tipos de investigación: la investigación sobre educación y la investigación en (desde) la educación. Describe la primera como la que realizan los investigadores no involucrados directamente en la práctica educativa en el aula y la segunda como la que desarrollan quienes están directamente implicados en el quehacer educativo, es decir, los docentes y directivos escolares, quienes con su trabajo pretenden cambiar su propia realidad, movidos por intereses ideológicos y académicos. El texto centra su atención en este último tipo de investigación y analiza algunas de sus características, entre ellas, su carácter político, la relación entre teoría y práctica, su perspectiva crítica, el dilema del método, el colectivo como sujeto de investigación y el rol del saber externo.
\end{abstract}

Palabras clave: Investigación en educación, práctica educativa, cambio escolar.

\begin{abstract}
The present reflection text approaches two investigation types: the investigation about education and the investigation in the education. The first one the way how the investigators not involved directly in the educational practice in the classroom do. And the second one is about the way how some investigators are implicated directly in the educational chore, that is the educational ones and school directive who seek to change their own reality with their work, moved by ideological and academic interests. The text centers its attention in this last investigation type and it analyzes some of its characteristics, among them, its political character, the relationship between theory and practice, its critical perspective, the dilemma of the method, the community as subject of investigation and the list of the external knowledge.
\end{abstract}

Key words: Investigation in the education, educational practice, scholastic change.

\footnotetext{
* Profesor Universidad Pedagógica Nacional. perez@colnodo.apc.org

** Texto presentado en el Panel sobre la relación entre innovación e investigación, en el V Congreso Distrital de Investigación e Innovación Educativa, organizado por el IDEP, en Bogotá. 2002.
} 
Me han invitado a hablar sobre la relación entre investigación de aula, innovación y calidad educativa. Esta casi imposible y compleja tarea puede ser abordada desde múltiples perspectivas. En aras de la brevedad que supone la intervención de veinte minutos en un panel, y por razones de interés académico, voy a delimitar el campo temático para referirme principalmente al primero de los componentes. Específicamente, quiero hablar de una tendencia particular de investigación en educación que considero está ganando fuerza en Latinoamérica, y que comienza a arrojar resultados en términos de transformaciones de la escuela. No sobra decir que hablaré desde mi propio recorrido como docente, como miembro de algunos colectivos de investigación en Colombia y desde una experiencia latinoamericana de cambio escolar en la que estoy participando actualmente. Desde allí, haré algunas afirmaciones y señalaré argumentos en favor de una perspectiva, una tendencia, mas no de un modelo. Valga decir que no tengo la pretensión de desarrollar el tema en su totalidad, sólo haré un acercamiento desde un ángulo particular.

\section{La investigación sobre educación y la investigación en educación}

Lawrence Stenhouse, un defensor de la investigación acción como alternativa de cambio en la escuela, hace una distinción clave entre dos tipos de investigación: investigaciones sobre la educación e investigaciones en (desde) la educación. Las investigaciones del primer tipo son aquellas realizadas por sujetos no involucrados directamente en la realidad que es objeto de investigación ${ }^{1}$. La segunda se refiere a aque-

\footnotetext{
1 "Los buenos profesores son necesariamente autónomos en el juicio profesional. No necesitan que se les diga lo que tienen que hacer. Profesionalmente, no dependen de investigadores, innovadores o inspectores. Esto no quiere decir que no estén abiertos a ideas creadas por otras personas en otros lugares y en otras ocasiones. Tampoco quiere decir que
}

Ila realizada directamente por los implicados desde el contexto mismo de su práctica profesional. La $\mathrm{OCDE}^{2}$ lo plantea como dos modelos: el modelo Olimpo, en el cual la investigación es cuestión de los científicos y no tiene por que contaminarse con la práctica, y el modelo Ágora, en el cual, en educación, teoría y práctica van unidas y se realimentan mutuamente.

Avancemos un poco más en la distinción. Al primer tipo pertenecerían, por ejemplo, las investigaciones de los psicólogos y sociólogos educativos, lingüistas, matemáticos, historiadores, antropólogos, músicos, administradores de la educación..., que toman la realidad escolar como objeto de análisis, y desde sus campos de trabajo, externos a la escuela, producen resultados de investigación: conocimientos, objetos didácticos, orientaciones curriculares, modelos comunicativos, secuencias de aprendizaje, formas de organización escolar... y las proponen para que sean apropiadas por las escue-

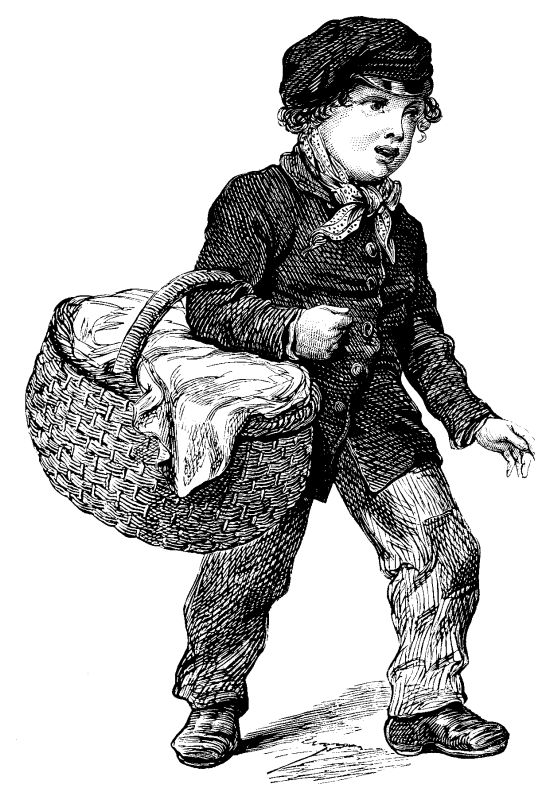

rechacen el consejo, la consulta o la ayuda", Stenhouse, Lawrence (1987), La investigación como base de la enseñanza, Madrid, Morata. 2 OCDE (1995): La Recherche et le développement en matiére d'enseignement, OCDE, Paris. las. El paradigma o modelo investigativo, así como los diseños metodológicos se definen, como en toda investigación, en función del objeto temático y el interés específico. Generalmente, este tipo de investigadores pertenece a un centro académico (universidad, instituto especializado, Ong), o se trata de equipos de expertos que se vinculan por intereses académicos o que se congregan alrededor de una convocatoria concreta, propuesta, generalmente, por una entidad financiadora. Estas investigaciones se orientan en atención a un sistema de intereses, principalmente académicos, particulares y hacen aportes al campo de discurso específico, previa legitimación por parte de la comunidad de expertos, legitimación que, dicho sea de paso, está ligada a la cultura de la escritura. Estos equipos de investigación, en la mayoría de los casos, cuentan con unas condiciones que permiten una alta dedicación de tiempos y esfuerzos. En el contexto latinoamericano, la mayoría de investigaciones está marcada por esta tendencia ${ }^{3}$.

Las investigaciones del segundo tipo, corresponden a aquellas adelantadas por los docentes o directivos docentes que toman como objeto de estudio su propia realidad escolar o un aspecto de la misma. Generalmente, este tipo de investigaciones pretende transformar dicha

\footnotetext{
3 "La mayoría de las innovaciones e investigaciones se han originado desde fuera de la escuela (81.35\%). De 193 proyectos registrados en 17 países, sólo 36 han surgido de la escuela". Blanco, Rosa y Mesina, Graciela (2000). Estado del arte de las innovaciones educativas en américa latina, Santiago, UNESCO.

"La universidad como institución moderna se ha configurado como espacio del saber. A las escuelas se debe llevar el discurso y de ellas se espera poder verificar que lo que piensa la academia es lo correcto. La pretensión de verdad y la voluntad de poder de la academia es resistente a encontrar saberes válidos en otros lugares... Quisiera pensar que las Ong y los maestros (investigación de aula) avanzan en este campo y que su significativa presencia en las convocatorias públicas del IDEP, se deba al reconocimiento de que la verdad está en otra parte" y Herrera, José Darío (2000), La investigación como práctica pedagógica, Bogotá, CAB.
} 
realidad, y los sujetos implicados se mueven por un sistema de intereses ideológicos, académicos y prácticos particulares; a su vez, la acción investigativa está determinada de manera fuerte por variables sociales y políticas de contexto.

Me quiero referir a una tendencia de investigación del segundo tipo, porque considero que es una alternativa que ha logrado transformaciones sostenidas en la cultura escolar en el ámbito latinoamericano. Me refiero a la investigación sobre la propia práctica.

\section{La investigación sobre la propia práctica, una alternativa de cambio}

Este tipo de investigaciones se nombra de diferentes formas, para simplificar, enunciémosla como investigación sobre la propia práctica. Se caracteriza por retomar como objeto privilegiado la práctica pedagógica de quienes hacen la investigación e implica una decisión explícita de compromiso con la transformación de la realidad existente, hecho que las diferencia de las investigaciones del primer tipo, en las cuales ésta no es la decisión subordinante. Esta decisión de base le otorga ciertas características y condiciones a la naturaleza misma de la investigación y a los sujetos que la adelan$\tan ^{4}$. Veamos algunas de estas características.

Carácter político. Además de asumir un compromiso explícito de cambio en la propia práctica, este tipo de investigación asume una perspectiva histórica e interpretativa,

\footnotetext{
${ }^{4}$ Estas investigaciones podemos encontrarlas referidas en modelos investigativos como la investigación acción, el análisis del discurso, la etnografía en educación, principalmente: "la etnografía del nosotros", como se nombra en México, y la pedagogía crítica; corrientes consolidadas en el ámbito de la investigación educativa. Esto también nos indica que lo que define a este tipo de investigación no es el paradigma o modelo investigativo sino su orientación a la transformación de una realidad y los sujetos que la desarrollan.
}

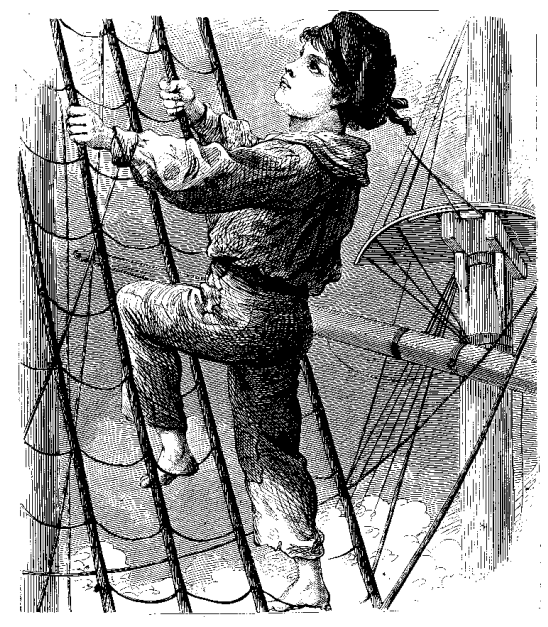

reactivo, sino como la posibilidad de análisis riguroso y profundo de las condiciones determinantes de la práctica propia, para de este modo contar con un contexto claro en el cual proponer una transformación. Condiciones como los dispositivos de distribución de los significados en el sistema educativo, los modos de legitimación de los saberes en la escuela, los formatos didácticos, los modelos comunicacionales y de asignación de la voz y el poder, la presencia de la multiplicidad cultural, los rituales escolares: rituales de apertura (cómo se inicia una sesión de clase), rituales de instrucción (yo explico, ustedes hacen silencio), rituales de refuerzo (la tarea..), rituales de resistencia (la copia...), rituales de verificación y control (la evaluación), rituales de cierre, son necesariamente objeto de reflexión crítica. De igual modo, una actitud crítica sobre la propia práctica pasa también por una análisis de los objetos y mediaciones que son empleados en la cultura escolar: los libros (sus funciones, el enfoque de su uso), los materiales no impresos (qué función pedagógica se asigna, en qué punto de la secuencia didáctica aparece...)... etcétera. De este modo vemos cómo esta perspectiva de investigación está cerca de las ideas claves de la pedagogía crítica enunciada por H. Giroux, P. Mac Laren o P. Freire.

El dilema del método. Dado que hay una decisión explícita de cambio y un reconocimiento de las condiciones de posibilidad del mismo, la investigación sobre la propia práctica se orienta hacia preguntas auténticas, formuladas desde el centro de la cultura escolar, de tal suerte que la naturaleza de la pregunta y el interés explícito de los sujetos de la investigación es lo que determina la decisión sobre el diseño y el enfoque investigativo por el que se opte. Así, es frecuente encontrar modelos cuantitativos al servicio de la investigación sobre la propia práctica, es el caso, por citar un ejemplo, de estudios sobre el pensamiento didáctico del profesor, o de estudios

\footnotetext{
5 Freire, Paulo.Pedagogía de la autonomía,

México: FCE, 1990.
}

Un perspectiva crítica. Este es otro factor clave, pero tomando el término no en el sentido contestatario y 
de análisis del discurso en el aula, donde se emplean modelos estadísticos de análisis semántico, pero la intención última es rastrear las concepciones que subyacen a la práctica pedagógica. $O$ en otros términos, la técnica está sobordinada al interés y a la pregunta de investigación.

Creo que en Latinoamérica se han montado muchos cursos inútiles de metodología de investigación, bajo el supuesto de que el dominio de una técnica hace al investigador; incluso por esta vía, se cree que es posible capacitar en investigación. Por supuesto que no estoy negando la validez y la importancia de los avances en modelos de investigación, pero creo que la vía de la instrucción en investigación no es pertinente porque subordina el interés investigativo al método. Creo que esta vía, en el mejor de los casos, puede conducir a formar buenos ayudantes de investigación, categoría de por sí problemática, por deficitaria y excluyente.

\section{El colectivo como sujeto de investigación}

Para concretar la intención de transformación, se requiere la existencia de un grupo permanente de docentes que define y delimita un proyecto de trabajo, e inicia el proceso de análisis de sus propias prácticas. Este equipo se ocupará del análisis de su realidad educativa, para desde allí identificar líneas de cambio y proyectos de investigación. En esta dinámica necesariamente existirán momentos en los que se requiera acompañamiento externo, con la claridad de que se trata de un interlocutor que va a contrastar lo que el colectivo va elaborando y encontrando y que desde su experiencia aporta ideas y orientaciones, tanto conceptuales como técnicas. Es probable que un equipo que se está iniciando requiera de mayor interlocución, y

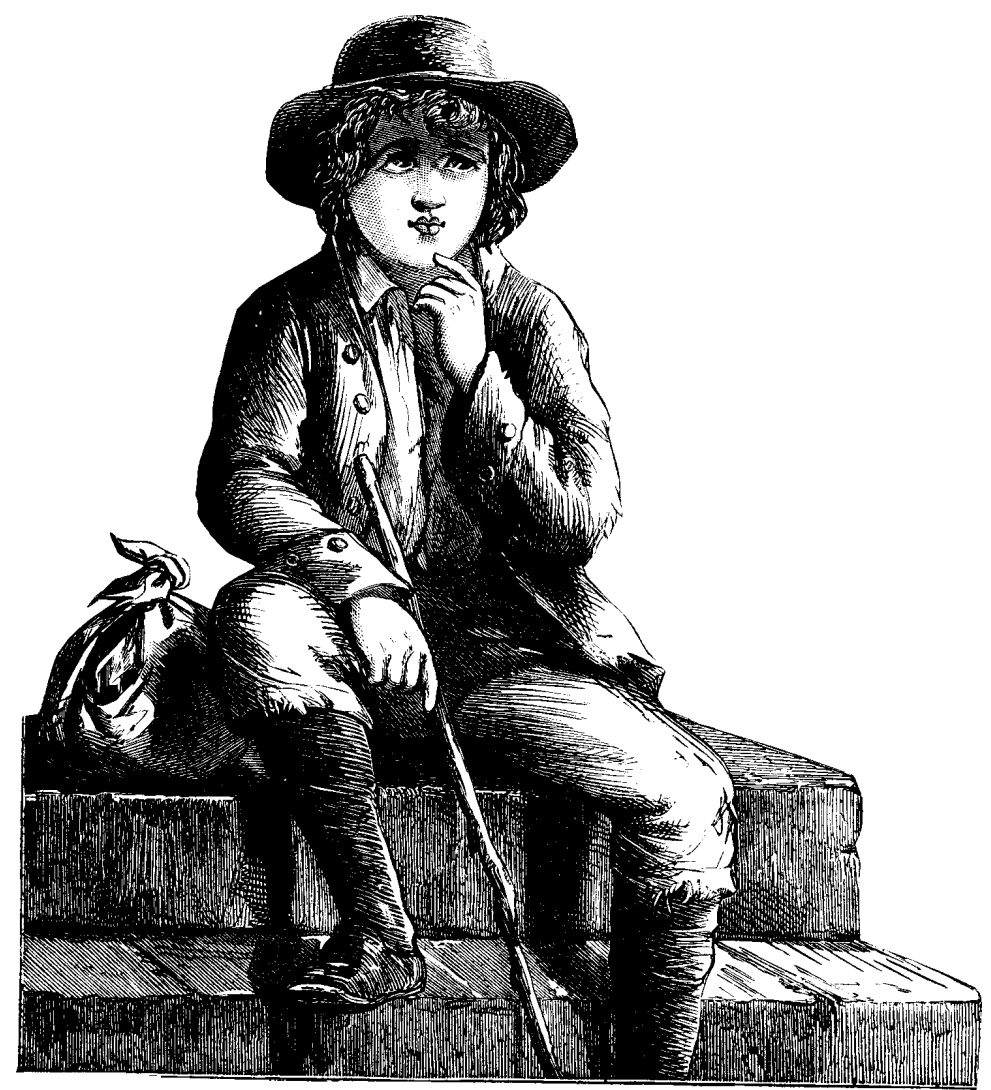

acompañamiento, cada dinámica será distinta, pero es necesario decir que la interlocución no puede generar dependencia; al contrario, debe orientarse hacia la producción de autonomía intelectual del equipo de base.

En la vía de conformarse como equipo investigador, la confrontación de los avances, tanto en el interior del colectivo, como fuera de él, a través de la escritura, juega un papel central. Es necesario avanzar hacia la producción de la escritura pública con todas las complejidades que esto implica. Se requiere contrastar lo logrado con lo que hacen otros en otros ámbitos. Esto indica que se requiere vincularse a una comunidad académica cada vez más amplia; ese es el horizonte. Considero que el Estado debe promover las condiciones para que esto ocurra; de hecho, creo que ese es el mérito del IDEP en la actualidad, y los equipos externos a la escuela deben hacer propuestas de acompañamiento que favorezcan la conformación de grupos autónomos.

El rol del saber externo. Un último elemento clave, que desarrollaré un poco más, es la función que cumple el saber externo, sea representado en sujetos: expertos o entidades que llegan a la escuela, o en discursos y textos (la teoría de vanguardia), o en experiencias ya probadas (las experiencias exitosas). En este punto, es necesario decir que la investigación sobre la propia práctica supone una relación de diálogo con el saber existente y con el experto, con las investigaciones del primer tipo, pero en la perspectiva de contrastar, confrontar, explorar, indagar, y no como una relación de dependencia.

Las investigaciones sobre la propia práctica, por su naturaleza misma, no pueden asumir la posición ingenua de "voy a aplicar esto a ver cómo me va...". En este caso hay, al menos, una ingenuidad implicada: que es posible hacerlo como otro lo hizo. Esto no es posible, porque para 
hacerlo necesitaría que mi pregunta investigativa fuera la misma de quien formuló la solución, lo que implicaría que mi sistema de intereses, expectativas, visiones del mundo fueran compartidos con él. Por otra parte, supondría que las concepciones sobre enseñanza, conocimiento, verdad, comunicación, que soportan la solución, fueran comparables, pues éstas son las condiciones de su puesta en marcha y para ir un poco más lejos, implicaría que los sujetos que acompañan mi práctica, los estudiantes, fueran comparables, y esto no es posible, ni siquiera en el marco de un mismo grupo etáreo y en condiciones socioeconómicas similares. Así que resulta, definitivamente, muy difícil instrumentar y operacionalizar una experiencia para ser replicada; además, este énfasis supone una posición problemática de quien lo promueve, pues implica una opción, en fin, instrumental, en vez de una opción de formación y cambio sustentado. O dicho en otra jerga, estaríamos de nuevo en una perspectiva deficitaria, al asumir que unos saben y otros hacen. De hecho, ese afán de promover la réplica, bienintencionado, se debe en muchos casos a necesidades administrativas y de recursos que vislumbran esta vía como económica y rápida. Pero no deja de ser ingenua.

Considero que esta tendencia no tiene en la actualidad ninguna vigencia, ni la ha tenido, pues si esto fuera posible, la tan nombrada "mala calidad educativa" ya sería un fantasma del pasado, pues bastaría identificar unas cuantas experiencias exitosas e instrumentar su replica. Pero la sociología de la educación se ha encargado de mostrarnos que una experiencia toma su carácter en atención a sus condiciones de posibilidad: sus condiciones de producción, de puesta en escena y de recepción. En fin, esa ingenuidad de que las experiencias son transferibles mecánicamente sólo quedan bien en una declaración de intenciones que no es posible aterrizar y que difícilmente se puede evaluar en su impacto.
Pero lo anterior no implica que no sea posible aprender de lo que ya ha sido probado en otros contextos. Al contrario, es necesario ver sus posibilidades y alcances. Por ejemplo, frente a una problemática específica identificada luego de un análisis crítico de la realidad escolar, se podría estudiar a fondo una alternativa o solución existente, analizar las ideas y teorías que la sustentan, evaluar sus condiciones y contexto de implementación, y tal vez el resultado sea que nos quedemos con unos criterios, unos conceptos y unas acciones que ayuden a definir nuestra propia perspectiva, o puede ocurrir que podamos retomar la experiencia completa, pues si, luego de su análisis, concluimos que cumple con nuestra expectativa, no desdibuja la perspectiva ni pedagógica ni disciplinar que queremos favorecer, y se adecúa al contexto propio, bienvenida la exploración crítica de la misma, que ya no se podrá llamar réplica, por tratarse de una decisión resultante de una actitud investigativa.

Incluso podría ocurrir que en esa exploración llegáramos un poco más allá del resultado encontrado en su versión original; esto ya es un resultado de investigación. Quiero insistir en que no se trata en absoluto que cada docente invente cada día una solución, que tal vez ya esté inventada, ni tampoco estoy defendiendo una posición conservadora y paralizante: "hacer sólo lo que ya funciona", es más bien una invitación a que la exploración de una alternativa sea asumida rigurosamente por los docentes como un ejercicio crítico, pero estoy seguro que debe revisarse lo existente antes de emprender una innovación o una investigación; de hecho, ésta es una condición de cualquier investigación.

Así que el enfoque de "hágalo usted mismo, yo le digo cómo", está en debate. Afortunadamente, el carácter crítico de la investigación sobre la propia práctica impide asumir este tipo de actitud mecanicista e ingenua.
Finalmente, debo decir que no he hablado de la innovación en este texto, pues considero que desde la perspectiva de la investigación sobre la propia práctica, no es posible innovar sin investigar.

Quiero cerrar mi intervención con unas palabras de Freire que creo sintetizan bien esta perspectiva de investigación: "No hay enseñanza sin investigación ni investigación sin enseñanza. Estos quehaceres se encuentran cada uno en el cuerpo del otro. Mientras enseño continúo buscando, indagando. Enseño porque busco, porque indagué, porque indago y me indago. Investigo para comprobar, comprobando intervengo, interviniendo educo y me educo. Investigo para conocer lo que aún no conozco y comunicar o anunciar la novedad... Hoy se habla con insistencia del profesor investigador. En mi opinión, lo que hay de investigador en el profesor no es una cualidad o una forma de ser o actuar que se agregue a la de enseñar. La indagación, la búsqueda, la investigación, forman parte de la naturaleza de la práctica docente"6.

\section{Bibliografía}

Blanco, Rosa y Mesina, Graciela. Estado del arte de las innovaciones educativas en américa latina, Santiago: UNESCO, 2000.

Freire, Paulo. Pedagogía de la autonomía, México: FCE, 1990.

Giroux, Henry y McLAren, Peter. Sociedad, cultura y educación. Buenos Aires: Niño y Dávila Editores, Instituto Freire, 1998.

Herrera, José Darío. La investigación como práctica pedagógica. Bogotá: CAB, 2000 .

OCDE, La Recherche et le Développement en Matiére D'enseignement, París: OCDE, 1995.

StenHOUSE, Lawrence. La investigación como base de la enseñanza. Madrid: Morata, 1987.

${ }^{6}$ Freire, Paulo, op. cit., p. 